\title{
Estrutura de populações de Rhodnius neglectus Lent e Psammolestes tertius Lent \& Jurberg (Hemiptera, Reduviidae) em ninhos de pássaros (Furnariidae) presentes na palmeira Mauritia flexuosa no Distrito Federal, Brasil
}

\author{
Rodrigo Gurgel-Gonçalves \& César A. C. Cuba
}

Laboratório de Parasitologia Médica e Biologia de Vetores, Faculdade de Medicina, Área de Patologia, Universidade de Brasília. 70910-900 Distrito Federal, Brasil. E-mail: rgurgel@ucb.br

\begin{abstract}
Population structure of Rhodnius neglectus Lent and Psammolestes tertius Lent \& Jurberg (Hemiptera, Reduviidae) in bird nests (Furnariidae) on Mauritia flexuosa palm trees in Federal District of Brazil. Rhodnius neglectus Lent, 1954 \{Rn\}and Psammolestes tertius Lent \& Jurberg, $1965\{\mathrm{Pt}\}$ are triatomines that occur in bird nests, mainly furnarid nests. Their biological cycles are known in laboratory conditions and few studies were done in wild ecotopes. To analyze the infestation and population structure of Rn and Pt in bird nests on Mauritia flexuosa Linnaeus palm trees in two climatic seasons we sampled 41 palm trees with Phacellodomus ruberVieillot, 1817 nests (22 in the wet season and 19 in the dry season) in four areas of Distrito Federal. The insects were collected by manual capture in the crown of palms, identified, separated by sex and immature stages. Faeces and salivary glands of Rn were examined to verify Trypanosoma cruzi Chagas, 1909 and/or T. rangeli Tejera, 1920 infection. Thirty five palm trees with $P$. ruber nests were infested by $\mathrm{Rn}(85 \%)$ and 22 by $\mathrm{Pt}(53 \%) .442$ insects were collected in the dry season (200 $\mathrm{Rn}$ and $242 \mathrm{Pt}$ ) and 267 in the wet season (136 Rn and $131 \mathrm{Pt}$ ). The area was the only factor related with triatomine density in palm trees. The population structure showed: a) higher abundance of adults in Pt populations, b) higher abundance of males in both species and, c) females laying eggs in both seasons. Within the 177 insects examined none was infected by $T$. cruzi or $T$. rangeli. The population structure of Rn and Pt were not significantly different in the wet and the dry season, suggesting no seasonality reproduction.
\end{abstract}

KEY WORDS. Arecaceae; Cerrado; Rhodniini; Triatominae.

RESUMO. Rhodnius neglectus Lent, 1954 \{Rn\}e Psammolestes tertius Lent \& Jurberg, 1965 \{Pt\} são triatomíneos que ocorrem em ninhos de aves, principalmente da família Furnariidae. O ciclo biológico dessas espécies é conhecido em condições de laboratório, sendo poucos estudos em ecótopos silvestres. Para analisar a infestação e estrutura de populações de $\mathrm{Rn}$ e Pt em ninhos de aves presentes na palmeira Mauritia flexuosa Linnaeus, em duas estações climáticas do Brasil Central, foram amostradas 41 palmeiras com evidências de nidificação de Phacellodomus ruber Vieillot, 1817 (22 na estação chuvosa e 19 na estação seca) em quatro áreas do Distrito Federal. Os insetos foram capturados usando-se coleta manual na copa da palmeira, identificados morfologicamente, separados por sexo e estádio ninfal. Fezes e glândulas salivares de Rn foram examinadas para verificar infecção por Trypanosoma cruzi Chagas, 1909 e/ ou T. rangeli Tejera, 1920. Trinta e cinco palmeiras com ninhos de $P$. ruber estavam infestadas por Rn (85\%) e 22 por Pt (53\%). 442 indivíduos foram coletados na estação seca (200 Rn e 242 Pt) e 267 na estação chuvosa (136 Rn e $131 \mathrm{Pt}$ ). O único fator relacionado significativamente com a densidade de triatomíneos nas palmeiras foi a área. A estrutura etária das populações mostrou: a) maior abundância de adultos nas populações de $\mathrm{Pt}, \mathrm{b})$ maior abundância de machos em ambas as espécies e c) presença de fêmeas ovipondo em ambas as estações. Nenhum dos 177 triatomíneos examinados estava infectado por $T$. cruzi ou $T$. rangeli. A estrutura etária das populações de Rn e Pt não diferiu significativamente entre as estações amostradas, indicando ausência de marcada sazonalidade para essas espécies.

PALAVRAS-CHAVE. Arecaceae; Cerrado; Rhodniini; Triatominae.

Rhodnius neglectus Lent, 1954 (Hemiptera, Reduviidae, Triatominae) é a espécie do gênero Rhodnius Stål, 1859 mais amplamente distribuída no Brasil, ocorrendo em 11 estados (Maranhão, Piauí, Pernambuco, Tocantins, Goiás, Bahia, Mi- nas Gerais, Mato Grosso, Mato Grosso do Sul, São Paulo e Paraná) e no Distrito Federal, entre 30 e 25으 de latitude sul, desde o nível do mar até cerca de 1000 m de altitude (LENT \& Wygodzinsky 1979, Silveira et al. 1984, Carcavallo et al. 1999). 
Populações dessa espécie ocorrem freqüentemente em palmeiras dos gêneros Attalea H.B. \& K., Acrocomia e Mauritia Linnaeus mas podem também ser encontradas em ninhos de pássaros de Furnariidae (Phacellodomus Reichenbach, 1853), e de mamíferos como Didelphis Linnaeus, 1758 (CARCAVAllo et al. 1998, Gurgel-Gonçalves et al. 2004). Porém, a invasão de R. neglectus no peridomicílio e até intradomicílio tem sido observada em Tocantins, Goiás, Minas Gerais, São Paulo e Paraná (BARretto 1979, Silveira et al. 1984, Garcia-Zapata et al. 1985, Guilherme et al. 2001, Silva et al. 2003), geralmente com baixos índices de infecção por Trypanosoma cruzi chagas, 1909, agente etiológico da doença de Chagas.

Psammolestes tertius Lent \& Jurberg, 1965 (Hemiptera, Reduviidae, Triatominae) é estritamente silvestre e tem sido encontrada em ninhos de pássaros da família Furnariidae, principalmente Phacellodomus rufifrons Wied-Neuwied, 1821, em diferentes estados no Brasil, com índices de infestação podendo al cançar até 100\% (LENT \& WYGODZINSKY 1979, SILVA \& LUSTOSA 1993). A espécie também pode ser encontrada em ninhos de outras aves como Anumbius annumbi Vieillot, 1817 e Mimus saturninus Lichtenstein, 1823 (CARCAVAlLo et al. 1998). Devido à forte associação com aves, P. tertius não tem relevância na transmissão do T. cruzi, embora já tenha sido observada a infecção natural e experimental (BARRETTO 1979).

Rhodnius neglectus e P. tertius ocorrem simultaneamente em ninhos de aves da espécie Phacel lodomus ruber (Passeriformes, Furnariidae) presentes na copa de Mauritia flexuosa Linnaeus (Arecaceae) (Gurgel-GonçAlves et al. 2004) (Fig. 1). Aspectos bionômicos dessas espécies como ciclo biológico, longevidade e estrutura etária são conhecidos em condições de laboratório (Perlowagora-Szumlewicz 1976, Mello 1977a,b, Forattinı et al. 1979, Heitzmann-Fontenelle 1984, Silva \& Lustosa 1993, Canale et al. 1999, Rocha et al. 2001), sendo poucos os estudos em ecótopos silvestres (Dıotaiutı \& Dias 1984, GuRgel-Gonçalves et al. 2004). O objetivo deste estudo é analisar a infestação e estrutura etária de populações de R. neglectus e P. tertius em ninhos de pássaros (P. ruber) presentes na palmeira M. flexuosa, em quatro áreas e duas estações climáticas do Distrito Federal (seca e chuvosa).

\section{MATERIAL E MÉTODOS}

O Distrito Federal está localizado entre as coordenadas

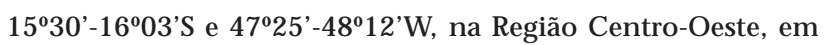
uma de suas áreas mais elevadas, o Planalto Central. As características ambientais da região estudada são: (I) $1000 \mathrm{~m}$ de altitude média, (II) precipitação média anual de $1545 \mathrm{~mm}$, (III) temperatura media anual de $21,1^{\circ}$ C , (IV) marcada sazonalidade, com uma estação seca e fria de maio a setembro (meses com precipitação menor que $100 \mathrm{~mm}$ ) e uma estação chuvosa e quente durante o resto do ano (RIBEIRO \& W WLTER 1998).

A pesquisa de triatomíneos foi realizada em formações savânicas chamadas "veredas", campos úmidos permanentes, colonizados por populações de palmeiras da espécie M. flexuosa e algumas espécies arbustivas. A amostragem das palmeiras foi

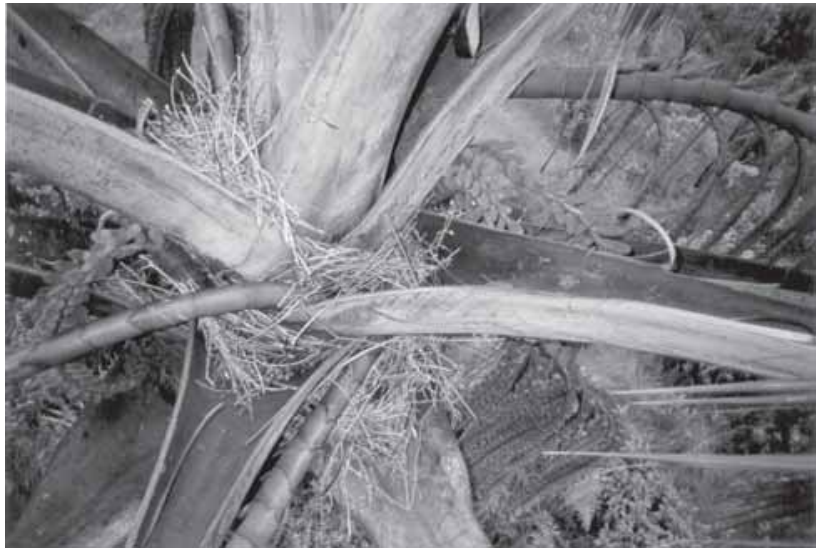

Figura 1. Ninhos de P. ruber na palmeira M. flexuosa, ecótopos de populações de P. tertius e R. neglectus em veredas no Distrito Federal.

feita em quatro veredas, localizadas na Estação Ecológica deÁguas Emendadas (1534'27"S, 4736'28"W), Reserva Ecológica do IBGE (1555' 54"S, 47ํ5'02"W), Colônia Agrícola Rajadinha (1546' 14"S, 47038'58"W) e Colônia Agrícola Alphaville - divisa Distrito Federal e Goiás (16004'05"S, 47ㅇ32'39"W). O método de coleta foi o mesmo usado por GURGEL-GonçALVES et al. (2004). As palmeiras foram escaladas e o material presente nas bainhas foliares (matéria orgânica, folhas e ninhos abandonados) foi coletado em sacos plásticos. Foram amostradas 41 palmeiras com evidências de nidificação de P. ruber (22 na estação chuvosa, fevereiro/ 2003; e 19 na estação seca, julho/2005). Na estação chuvosa a temperatura média varia entre 20 e $22 \circ \mathrm{C}$, com umidade relativa do ar entre $70 \%$ e $85 \%$. Julho é o mês mais frio e seco no Distrito Federal, com temperatura média entre 16 e 18ㄷ $\mathrm{C}$ e umidade relativa do ar podendo alcançar valores menores que 20\% (SILVAJúnIOR et al. 1998).

Os triatomíneos presentes nos ninhos no momento da coleta e, posteriormente, na triagem do material coletado em laboratório, foram capturados, separados por sexo (adultos), estádio ninfal (imaturos) e contabilizados. A identificação morfológica em nível de espécie foi feita usando as chaves descritas por LENT \& WYGODZINSKY (1979). Fezes e glândulas salivares de R. negl ectus foram examinadas para verificar infecção por Trypanosoma cruzi e/ou $\mathrm{T}$. rangeli.

Para comparar a infestação e estrutura populacional das espécies nas áreas e estações climáticas foram utilizadas as seguintes variáveis: a) número total de triatomíneos, b) número de triatomíneos/palmeira (densidade), c) índice adulto/ninfa ( $\mathrm{n}$ - total de adultos/ $\mathrm{n}$ total de ninfas), d) índice macho/fêmea ( $\mathrm{n}$ - total de machos/ $\mathrm{n}$ o total de fêmeas) e e) número médio de triatomíneos em cada estádio de desenvolvimento nas quatro áreas estudadas. O número total de triatomíneos de cada espécie capturado em uma mesma estação climática foi comparado usando-se teste qui-quadrado $\left(\chi^{2}\right)$. Para analisar a variação do número de triatomíneos por palmeira entre espécies, áreas e

Revista Brasileira de Zoologia 24 (1): 157-163, março 2007 
estações foi utilizada uma Anal ise de Variância (ANOVA) fatorial após transformação dos dados (raiz quadrada), com nível de significância de $p<0,01$. Testes paramétricos (teste-t) e não paramétricos (teste de Mann-Whitney U) também foram utilizados para comparação de médias, com nível de significância de $p<0,01$ (ZAR 1999). O cálculo dos testes foi realizado com o programa Statistica (STATSOFT 1996).

\section{RESULTADOS}

Considerando as quatro áreas amostradas, 35 palmeiras com ninhos de Phacellodomus ruber estavam infestadas por Rhodnius neglectus (85\%) e 22 por Psammolestes tertius (53\%). Rhodnius neglectus foi a espécie mais freqüente nos ninhos em ambas as estações (Fig. 2). Foram coletados 442 triatomíneos na estação seca (200 R. neglectus e 242 P. tertius) e 267 na estação chuvosa (136 R. neglectus e 131 P. tertius). Não foi observada diferença significativa comparando-se o número total de R. neglectus e $P$. tertius capturados em cada estação $\left(\chi^{2}=2,16\right.$; $\mathrm{p}=0,14)$. O número de triatomíneos/palmeira (densidade) variou bastante com médias de 12,7 $\pm 22,3$ (seca) e 5,9 $\pm 7,7$ (chuvosa) para P. tertius e 10,5 $\pm 11,7$ (seca) e 7,6 \pm 6,7 (chuvosa) para R. neglectus.

Dos três fatores analisados (área, estação climática e espécie) apenas a área influenciou significativamente a densidade de triatomíneos (ANOVA $F_{3,51}=6,8 ; p<0,01$ ), com Colônia Agrícola Rajadinha apresentando maiores valores (Fig. 3). Não foi observada diferença significativa comparando a densidade média de $P$. tertius e R. neglectus nas palmeiras (teste- $t_{55}=-1,58$; $p=0,12$ ). Apesar de um maior número de triatomíneos ter sido capturado na estação seca, não foi observada diferença significativa comparando a densidade média de triatomíneos em cada estação climática (teste- ${ }_{55}=1,30 ; p=0,20$ ).

Foi observado maior número de ninfas nas populações de R. neglectus em ambas as estações, com índice adulto/ninfa médio menor que 1 (Fig. 4). Apesar do número total de ninfas ter sido menor no na estação chuvosa, não foi observada diferença significativa comparando-se a média do índice adulto/ ninfa observada nas estações para essa espécie de triatomíneo (teste de Mann-Whitney $U=102,5 ; p=0,14$ ). No caso de $P$. tertius, uma maior quantidade de adultos foi observada na estação chuvosa com uma média de 1,74 adultos por ninfa (Fig. 4). A média observada na estação seca foi menor $(0,38)$, contudo essas diferenças não foram significativas (teste de MannWhitney $U=19,5 ; p=0,07$ ).

Ambas as populações apresentaram mais machos que fêmeas nas duas estações (Fig. 5), com índice macho/fêmea médio de 3,0 (seca) e 2,2 (chuvosa) para R. neglectus e 2,1 (seca) e 1,6 (chuvosa) para P. tertius nas áreas amostradas. Foram observadas fêmeas ovipondo em ambas as estações indicando eventos reprodutivos em Julho e Fevereiro.

A comparação da estrutura etária das populações de R. neglectus nas duas estações amostradas indicou pequenas diferenças no número de estádios iniciais ( 1 e 2) e adultos (machos

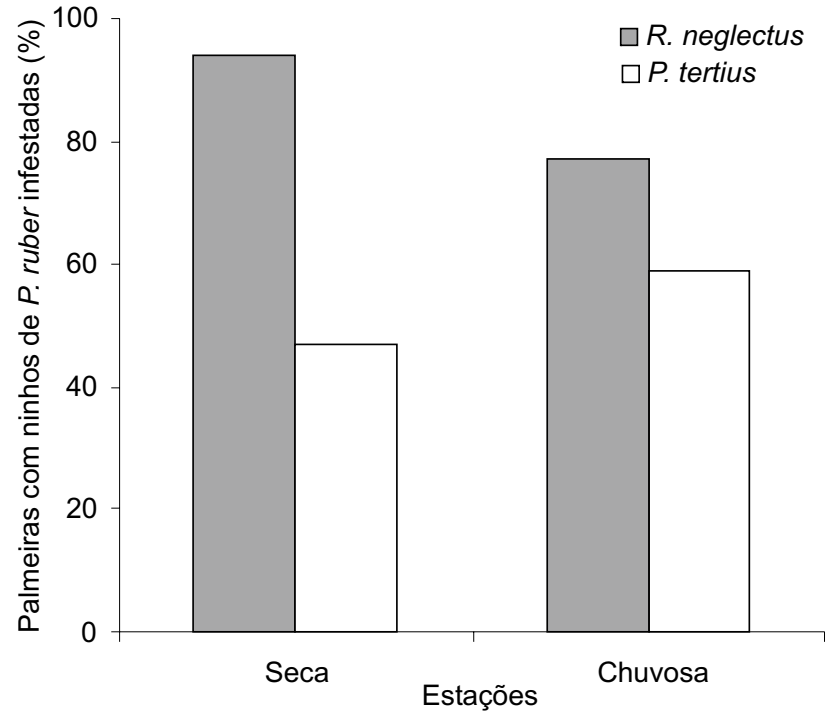

Figura 2. Porcentagem de palmeiras com ninhos de P. ruber infestadas por R. neglectus e P. tertius na estação seca e chuvosa no Distrito Federal.
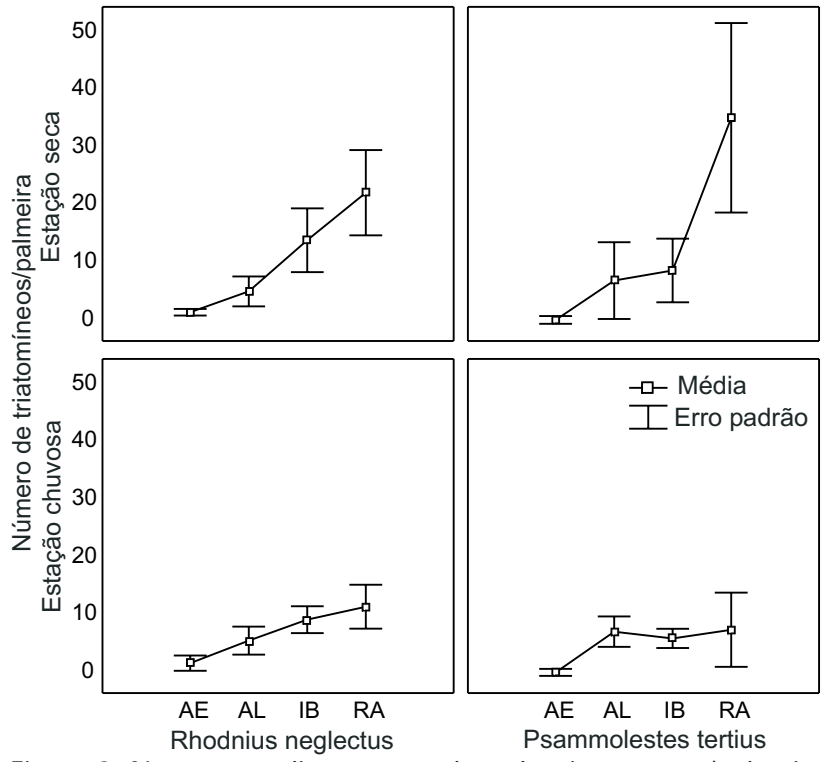

Figura 3. Número médio e erro padrão de triatomíneos/palmeira de acordo com a espécie (R. neglectus e P. tertius), área (AE: Estação Ecológica Águas Emendadas, AL: Colônia Agrícola Alphaville, IB: Reserva Ecológica do IBGE e RA: Colônia Agrícola Rajadinha) e estação climática (chuvosa e seca) no Distrito Federal.

e fêmeas); e uma maior variação no número de estágios intermediários (3, 4 e 5). Nenhuma dessas diferenças foi estatisticamente significativa (Fig. 5). No caso de P. tertius, houve pequena variação na abundância dos estádios ninfais e adultos entre 


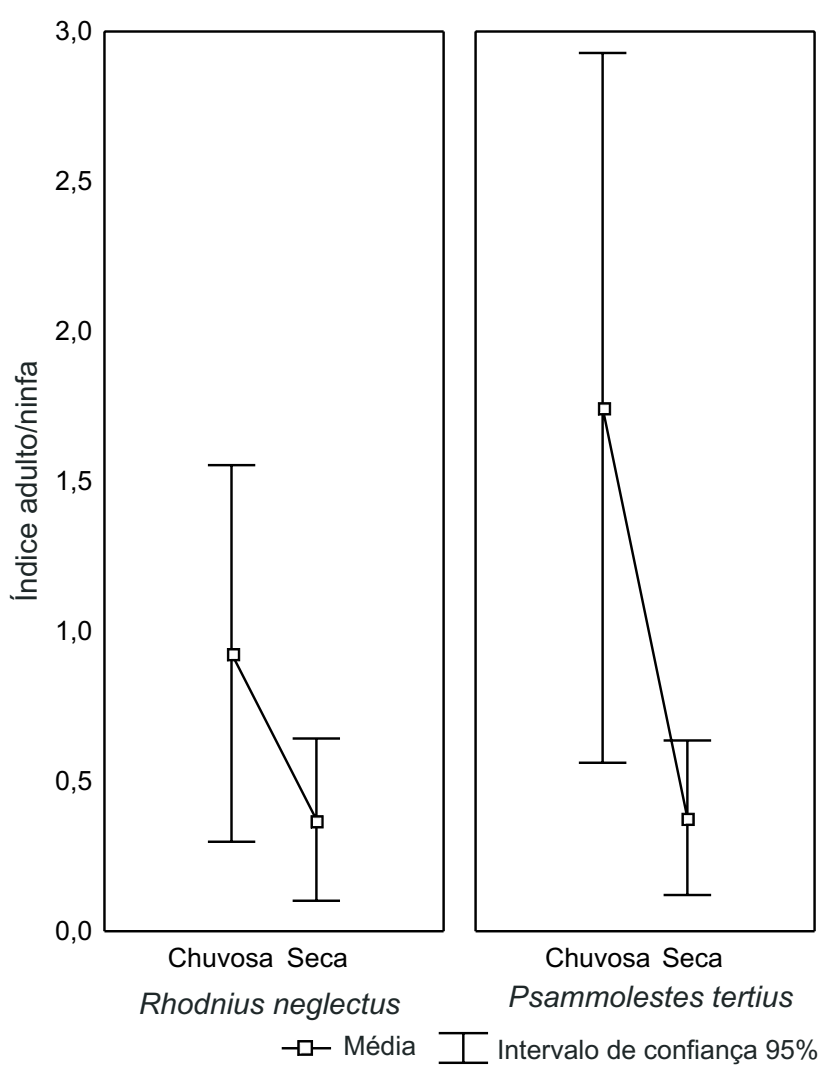

Figura 4. Média e intervalo de confiança (95\%) do índice adulto/ ninfa para R. neglectus e P. tertius em duas estações climáticas (chuvosa e seca) no Distrito Federal.

as estações (Fig. 5), sem diferenças significativas ( $p<0,01$ ). Também não foram observadas diferenças significativas comparando-se a estrutura etária de R. neglectus e P. tertius em cada estação. Nenhum dos 177 triatomíneos examinados (R. neglectus) estava infectado por $\mathrm{T}$. cruzi ou $\mathrm{T}$. rangeli.

\section{DISCUSSÃO}

Estudos relacionando abundância de insetos e sazonalidade no Cerrado mostram que os hemípteros apresentam maior abundância na estação chuvosa (PInHeIRo et al. 2002). Resultados com populações de R. neglectus em palmeiras do gênero Acrocomia também mostram que há um crescimento das colônias no verão (estação chuvosa), porém apontam a presença de ovos em menor quantidade no inverno seco (DIOTAIUTI \& DIAS 1984). No presente trabalho, a abundância de R. neglectus foi maior na seca (62\% dos indivíduos coletados) e fêmeas reprodutivas foram observadas em ambas as estações, indicando mais de um evento reprodutivo por ano. Além disso, não foram observadas diferenças na estrutura etária dessa espécie comparando-se ambas as estações estudadas (Fig. 5). Esses resultados sugerem então que R. neglectus pode apresentar ciclo reprodutivo contínuo durante o ano. No caso de P. tertius, apesar da maior abundância relativa de adultos ter sido observada na estação chuvosa, não houve diferença significativa na abundância dos estádios ninfais (ninfa 1 a ninfa 5) e adultos (machos e fêmeas) entre as estações climáticas (Fig. 5). Na Argentina, a presença de Psammolestes coreodes Bergroth, 1911 em ninhos de Furnariidae (P. ruber e Anumbius annumbi) também foi semelhante entre as estações climáticas (BAR et al. 1999). Esses resultados reforçam a hipótese de ausência de marcada sazonalidade para essas espécies de triatomíneos.

É conhecido que fatores climáticos podem afetar a duração do ciclo biológico, a distribuição, a dispersão pelo vôo, o número de repastos e até a probabilidade de transmissão de $T$. cruzi pelos triatomíneos (CuRTO dE CASAS et al. 1999). Os ciclos biológicos de P. tertius e R. neglectus em condições de laboratório são muito rápidos em comparação a outras espécies de Triatominae, variando de 81 a 225 dias (R. neglectus) e de 124 a 165 dias (P. tertius), dependendo das condições de temperatura e umidade relativa (CAnale et al. 1999). Para R. neglectus, Rocha et al. (2001) mostram nítida correlação entre temperaturas mais altas e diminuição do período embrionário e período médio de desenvolvimento ninfal em condições delaboratório. Entretanto, osmesmos autores apontam que temperaturas muito elevadas podem inviabilizar a sobrevivência das colônias, devido à alta mortalidade associada. Porém, em condições naturais, a influência das variações de temperatura e umidade do ambiente pode ser minimizada para as espécies de triatomíneos que vivem em palmeiras. Segundo LORENZO et al. (2003), a variação de temperatura e umidade na base das folhas das palmeiras é menor quando comparada com o ambiente externo, favorecendo o desenvolvimento das colônias silvestres em condições climáticas mais estáveis. Além disso, a diferença de temperatura média entre a estação chuvosa e seca não é tão grande na área estudada (SıLVA-JúnıoR et al. 1998). Dessa forma, haveria a possibilidade de ocorrer dois ou até três ciclos biológicos anuais contínuos para R. neglectus e P. tertius, dependendo da disponibilidade de fonte alimentar na palmeira.

Segundo Carcavallo \& Tonn (1976), a frequência de alimentação influencia bastante o ciclo biológico das espécies de triatomíneos. Colônias de R. prolixus freqüentemente alimentadas mostraram ciclos biológicos mais curtos em relação às colônias alimentadas em interval os de tempo maiores. Além disso, o tipo de sangue também pode influenciar o ciclo biológico dos triatomíneos. DıOTAIUTı \& DiAs (1987) mostraram que colônias de R. neglectus alimentadas com camundongos tinham períodos mais curtos de desenvolvimento quando comparadas com colônias alimentadas com pombos. Apesar de não ter sido obtida uma evidência direta da fonte al imentar de R. neglectus e P. tertius, essas espécies de triatomíneos devem se alimentar freqüentemente do sangue de $P$. ruber, espécie de pássaro mais comum na copa de M. flexuosa (GURGEL-GonçALVES et al. 2004). A ausência de infecção de T. cruzi e T. rangeli em R. neglectus observada neste trabal ho também indica pouco contato dessa espécie com sangue de mamíferos. 


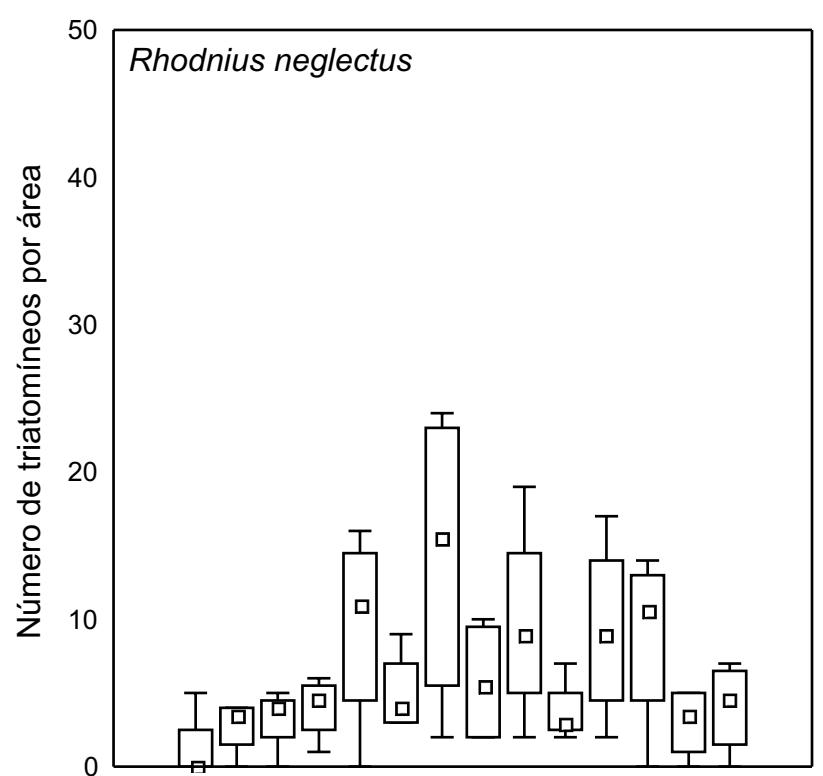

1S 1 C 2S 2C 3S 3C 4S 4C 5S 5CMSMCFS FC

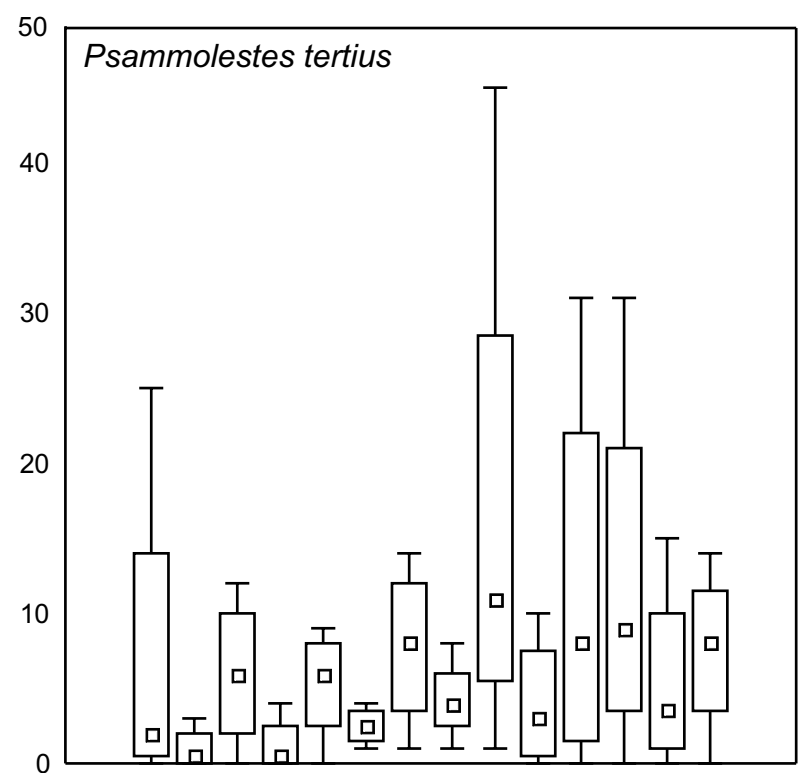

1S $1 \mathrm{C} 2 \mathrm{~S} 2 \mathrm{C} 3 \mathrm{~S} 3 \mathrm{C} 4 \mathrm{~S} 4 \mathrm{C} 5 \mathrm{~S} 5 \mathrm{C}$ MSMC FS FC Estádios de desenvolvimento em cada estação climática

口 Mediana $\square$ 25-75\% percentil 工 Mín-Máx

Figura 5. Variação do número de triatomíneos por área em cada estádio de desenvolvimento de R. neglectus (esquerda) e P. tertius (direita) presentes em ninhos de P. ruber na palmeira M. flexuosa na estação chuvosa (C) e seca (S) no Distrito Federal. Os diagramas de caixas mostram a mediana, o percentil (25-75\%) e os valores mínimos e máximos de cada estádio. (1-5) estádios ninfais, (M) macho, (F) fêmea. Em nenhum dos casos houve diferença significativa entre o número médio de insetos na seca e na chuva considerando $p<0,01$.

Algumas características biológicas de pássaros do gênero Phacellodomus podem favorecer o bom desenvolvimento das colônias de triatomíneos como: a) ninhos grandes, em média com duas a três câmaras de incubação de filhotes, b) formação de territórios com até 10 indivíduos que usam os ninhos durante todo o ano para dormir, incubar os ovos e criar seus fiIhotes, c) permanência dos filhotes no território dos pais por até 16 meses, os quais ajudam na alimentação de outros filhotes em um complexo sistema social, d) reutilização dos mesmos ninhos para reprodução (SICK 1997, CARRARA \& RodrigueS 2001). Além disso, em P. rufifrons (espécie filogeneticamente próxima a $\mathrm{P}$. ruber) foi observada alta assincronia, com grupos de pássaros iniciando postura tanto no início quanto no final da estação chuvosa (CARRARA \& Rodrigues 2001). Se isso também ocorrer com $P$. ruber, seria mais um argumento a favor da ausência de padrão sazonal para as espécies de triatomíneos na copa de M. flexuosa e o maior número de triatomíneos capturado na seca neste estudo poderia estar relacionado com uma maior abundância de fontes alimentares (adultos e filhotes de P. ruber) em julho de 2005. Finalmente, de acordo com GuRGELGonçALVES et al. (2004) a existência de ninhos de aves (principalmente $P$. ruber) nas bainhas foliares das palmeiras sugere ser uma das principais características que determinam uma maior abundância de R. neglectus e P. tertius em M. flexuosa. A varia- ção da densidade de triatomíneos por palmeira e por área encontrada no presente estudo, sendo mais abundantes na Colônia Agrícola Rajadinha (principalmente para P. tertius), poderia ser explicada pelo tamanho e maior quantidade dos ninhos de P. ruber em M. flexuosa nessa área. Em ninhos pequenos ou muito velhos não foram obtidos triatomíneos, diferentemente dos ninhos grandes e com evidências da presença do pássaro (penas novas, filhotes, casca de ovos), onde foram obtidas grandes colônias de R. neglectus e P. tertius (Fig. 1).

O predomínio de ninfas de R. neglectus (Fig. 4) está de acordo com a estrutura etária geralmente observada em populações de Rhodnius em palmeiras, com índice adulto/ninfa inferior a 1,00 variando entre 0,36 e 0,90 (PIZARRo \& Romaña 1998). Isso pode ser explicado pela dispersão dos insetos, abandonando o ninho após chegarem à idade adulta. Já P. tertius mostrou um menor número de ninfas na estação chuvosa (índice adulto/ninfa: 1,74), resultado semelhante ao obtido por SILVA \& LustoSA (1993) em ninhos de P. rufifrons em árvores do Cerrado (índice adulto/ninfa: 1,46 ) no início das chuvas. Isso pode estar relacionado com uma maior longevidade dos adultos sobreviventes de gerações anteriores que não dispersaram, com uma menor taxa de reprodução e/ou uma maior mortalidade de ninfas durante as chuvas de verão. O predomínio de machos de ambas as espécies nos ninhos (Fig. 5) estaria relaciona- 
do com a maior sobrevivência média ao jejum dos machos $(41,7$ dias) quando comparada com a de fêmeas (33,4 dias) (SıLva \& LUSTOSA 1993). Outro fator poderia ser a maior capacidade de dispersão das fêmeas, que deixariam as palmeiras para se reproduzirem em outro local, porém, existem poucos estudos sobre padrões de dispersão de triatomíneos (CARCAVALLo \& Tonn 1976).

Outro fator que poderia estar influenciando as diferenças observadas seria o método de coleta, subestimando a população de triatomíneos nos ninhos presentes em M. flexousa. Uma limitação seria a perda de ninfas 1 no momento da coleta, por serem muito pequenas e mais difíceis de serem visualizadas. Porém, as coletas foram padronizadas e realizadas pelo mesmo pesquisador nas duas estações climáticas e nas mesmas áreas. Finalmente, um estudo longitudinal, realizando coletas com um mesmo esforço amostral mensal (cerca de 20 palmeiras com ninhos de P. ruber) poderia avaliar de forma mais precisa a estrutura populacional de ambas as espécies de triatomíneos, porém, o impacto sobre as populações do pássaro deve ser consideravelmente grande devido à remoção dos ninhos para análise.

O ciclo evolutivo, a fecundidade, fertilidade e sobrevivência ao jejum de P. tertius e R. neglectus são similares em condições de laboratório (Silva \& LustosA 1993), entretanto, em condições naturais, o comportamento biológico e as estratégias de dispersão dessas espécies devem ser diferentes: R. neglectus apresentando maior potencial de dispersão (maior freqüência nos ninhos de P. ruber) e maior ecleticismo alimentar, visto que pode ser encontrada em outros tipos de ninhos presentes em M. flexuosa (Gurgel-GonçAlves et al. 2004) e P. tertius apresentando maior potencial de colonização nos ninhos de P. ruber (maior densidade de triatomíneos/palmeira) e aparentemente não sendo encontrada associada a outros tipos de ninhos em M. flexuosa. Em ambas as espécies a estrutura etária das populações não diferiu significativamente entre as estações amostradas, sugerindo que não há marcada sazonalidade para esses triatomíneos na copa de M. flexuosa, sendo o desenvolvimento das colônias mais dependente da biologia da fonte alimentar, no caso, o pássaro P. ruber.

\section{AGRADECIMENTOS}

Ao CNPq e FAP/DF pelo apoio financeiro. Aos estagiários Fábio e Welber pela colaboração no trabalho de campo; aos pesquisadores Alexandre R.T. Palma (Universidade Católica de Brasília), José Jurberg (Fiocruz), Raúl A. Laumann (Universidade Católica de Brasília e Embrapa Recursos Genéticos e Biotecnologia) e revisores anônimos pelas críticas e sugestões.

\section{REFERÊNCIAS BIBLIOGRÁFICAS}

Bar, M.E.; M.P. Damborsky; B.M. Alvarez; E.B. Oscherov \& S.M. MAZzA. 1999. Triatominos silvestres detectados en nidos de aves de al gunos departamentos de la provincia de Corrientes, Argentina. Revista de la Sociedad Entomológica Argentina 58 (3/4): 43-50.

Revista Brasileira de Zoologia 24 (1): 157-163, março 2007
BarRetto, M.P. 1979. Epidemiologia, p. 89-291. In: Z. BREneR \& Z.A. Andrade (Eds) Trypanosoma cruzi e Doença de Chagas. Rio de Janeiro, Guanabara Koogan, 463p.

Canale, M.D.; J. Jurberg; R.U. Carcavallo; C. Galvão; I. Galíndez Giron; C.A. Mena Segura; D.S. Rocha \& A. Martinez. 1999. Bionomics of some species, p. 839-890. In: R.U. Carcavallo; I. Galíndez Girón; J. JuRberg \& H. Lent (Eds). Atlas of Chagas Disease vectors in Americas. Rio de Janeiro, Fiocruz, vol. 3, 471p.

Carcavallo, R.U. \& R.J. Tonn. 1976. Rhodnius prolixus Stal, 1859, p. 209-219. In: R.U. Carcavallo; J.E. Rabinovich \& R.J. Tonn (Eds). Factores biológicos y ecológicos en la enfermedad de Chagas. Tomo I. Epidemiologia - vectores. Buenos Aires, Organización Panamericana de la Salud, OMS, 250p.

Carcavallo, R.U.; M.E.F. Rodríguez; R.Salvatella; S.I. Curto de Casas; I. Sherlock; C. Galvão; D.S.Rocha; I. Galíndez Girón; M.A.O Arocha; A. Martinez; J.A. da Rosa; D.M Canale; T.H. FARR \& J.M.S. BARATA. 1998. Habitat and related fauna, p. 561-600. In: R.U. Carcavallo; I. Galíndez Girón; J. Jurberg \& H. LENT (Eds). Atlas of Chagas Disease vectors in Americas. Rio de Janeiro, Fiocruz, vol. 2, 326p.

Carcavallo, R.U.; S.I. Curto de Casas; I. Sherlock; I. Galíndez Girón; J. Jurberg; C. Galvão; C.A. Mena Segura \& F. Noireau. 1999. Geographical distribution and alti-longitudinal dispersion, p. 747-792. In: R.U. Carcavallo; I. Galíndez Girón; J. JuRberg \& $\mathrm{H}$. LeNT (Eds). Atlas of Chagas Disease vectors in Americas. Rio de Janeiro, Fiocruz, vol. 3, 471p.

Carrara, L.A. \& M. Rodrigues. 2001. Breeding biology of the rufous-fronted thornbird Phacellodomus rufifrons, a Neotropical ovenbird. International Journal of Ornithology 4 (3/ 4): 209-217.

Curto de Casas, S.I.; R.U.Carcavallo; I. Galíndez Girón \& J.J Burgos. 1999. Bioclimatic factors and zones of life, p. 793-838. In: R.U. Carcavallo; I. Galíndez Girón; J. Jurberg \& H. Lent (Eds). Atlas of Chagas Disease vectors in Americas. Rio de Janeiro, Fiocruz, vol. 3, 471p.

DıotAIUTI, L. \& J.C.P. DıAs. 1984. Ocorrência e biologia de Rhodnius neglectus, Lent, 1954 em macaubeiras da periferia de Belo Horizonte, Minas Gerais. Memórias do Instituto Oswaldo Cruz 79 (3): 293-301.

Dıotalutı, L. \& J.C.P. DiAs. 1987. Estudo comparativo do ciclo evolutivo de Rhodnius neglectus alimentados em pombos e camundongos. Revista da Sociedade Brasileira de Medicina Tropical 20: 95-100.

Garcia-Zapata, M.T.; D. Virgens; V.A. Soares; A. Bosworth \& P.D. MARSDEN. 1985. House invasion by secondary triatominae species in Mambaí, Goiás-Brazil. Revista da Sociedade Brasileira de Medicina Tropical 18 (3): 199-201.

Guilherme, A.L.; G.C. Pavanelli; S.V Silva; A.L. Costa \& S.M. de Araújo. 2001. Secondary triatomine species in dwellings and other nearby structures in municipalities under epidemiological surveillance in state of Parana, Brasil. Revista Panamericana de Salud Publica 9 (6): 385-392. 
Gurgel-Gonçalves, R.; M.A. Duarte; E.D. Ramalho; C.A. Romaña \& C.A.C. CuBA. 2004. Distribuição espacial de populações de Triatominae (Hemiptera, Reduviidae) em palmeiras da espécie Mauritia flexuosa no Distrito Federal, Brasil. Revista da Sociedade Brasileira de Medicina Tropical 37 (3): 241 247.

Forattini, O.P.; O.A. Ferreira, E.O. Rocha e Silva \& E.S. Rabello. 1979. Aspectos ecológicos da tripanossomíase americana. XV - Desenvolvimento, variação e permanência de Triatoma sordida, Panstrongylus megistus e Rhodnius neglectus em ecótopos artificiais. Revista de Saúde Pública 12: 220-234.

Heitzmann-Fontenelle, T.J. 1983/1984. Bionomia comparativa de triatomíneos. VII - Rhodnius neglectus Lent, 1954 (Hemiptera, Reduviidae). Memórias do Instituto Butantan 47/48: 183188.

LENT, H. \& P.WYGodZINSKY. 1979. Revision of the Triatominae (Hemiptera, Reduviidae), and their significance as vectors of Chagas Disease. Bulletin American Museum of Natural History 163: 520-529.

Lorenzo, M.G.; S.A. Minoli; C.R. Lazzari; A.S. Paula \& Diotaiutı, L. 2003. Microclima dos ecótopos naturais das espécies do gênero Rhodnius no município de Tocantinópolis, estado de Tocantins, Brasil. Revista da Sociedade Brasileira de Medicina Tropical 36 (Supl. I): 425.

Melto, D.A. 1977a. Biology of Triatominae (Reduviidae, Hemiptera) from north of Formosa county (Goiás-Brazil). II Length of life cycle of Rhodnius neglectus Lent 1964. Revista da Sociedade Brasileira de Medicina Tropical 11: 63-66.

Mello, D.A. 1977b. Biology of Triatominae (Reduviidae, Hemiptera) from north of Formosa county (Goiás-Brazil). III Length of life cycle of Psammolestes tertius Lent \& Jurberg 1965. Revista da Sociedade Brasileira de Medicina Tropical 11: 147-149.

Perlowagora-Szumlewicz, A. 1976. Laboratory colonies of Triatominae, biology and population dynamics.Pan American Health Organization Scientific Publication 318: 63-82.

Pinheiro, F.; I.R. Diniz; D. Coelho \& M.P.S. Bandeira. 2002.
Seasonal pattern of insect abundance in the Brazilian Cerrado. Austral Ecology 27 (2): 132-136.

PizarRo, J.C. \& C.A. Romaña. 1998. Variación estacional de una población silvestre de Rhodnius pallescens Barber, 1932 (Heteroptera: Triatominae) en la costa caribe Colombiana. Bulletin de L'Institut français d'études andines 27 (2): 309-325.

Ribeiro, J.F. \& B.M.T. Walter. 1998. Fitofisionomias do bioma Cerrado, p. 87-167. In: S.M. SAno \& S.P. Almeida (Eds). Cerrado Ambiente e Flora. Planaltina, Embrapa, 556p.

Rocha, D. S.; J. Jurberg; R.U. Carcavallo; V. Cunha \& C. Galvão. 2001. Influência da temperatura e umidade no desenvolvimento de Rhodnius neglectus Lent, 1954 em laboratório (Hemiptera, Reduviidae, Triatominae). Revista da Sociedade Brasileira de Medicina Tropical 34 (4): 357-363.

Sick, H. 1997. Ornitologia Brasileira. Rio de Janeiro, Editora Nova Fronteira, 862p.

Silva, I.G. DA \& E. DE S. Lustosa. 1993. Biologia de Psammolestes tertius Lent \& Jurgberg, 1965 (Hemiptera, Reduviidae). Revista Patologia Tropical 22 (1): 29-42.

Silva, R.A.; S.A.S. Scandar; S.M.P. Sampaio; C. Pauliquevis Júnior \& V.L.C.C. Rodrigues. 2003. Programa de controle de doença de Chagas (PCDCH): Rhodnius neglectus (Lent, 1954) (Hemiptera, Reduviidae, Triatominae) no estado de São PauIo, Brasil. Revista da Sociedade Brasileira de Medicina Tropical 36 (Supl. 1): 395.

Silva-Júnior, M.C.; J.M. Felfili; P.E. Nogueira \& A.V. Resende. 1998. Análise Florística das matas de galeria no Distrito Federal, p. 51-84. In: J.F. Ribeiro (Ed). Cerrado matas de galeria. Planaltina, Embrapa, 164p.

Silveira, A.C.; V.R. Feitosa \& R. Borges. 1984. Distribuição de triatomíneos capturados no ambiente domiciliar, no período de 1975/83, Brasil. Revista Brasileira de Malariologia e Doenças Tropicais 39: 15-312.

StatSoft InC. 1996. Statistica for Windows. Tulsa, Computer program manual.

ZAR, J.H. 1999. Biostatistical analysis. Upper Saddle River, Prentice-Hall, $2^{\text {nd }}$ ed., 663p.

Recebido em 27.VI.2006; aceito em 06.III.2007. 